\title{
On the Number of Vertices of the Convex Hull of Random Points in a Square and a Triangle
}

By

\section{Christian Buchta}

(Vorgelegt in der Sitzung der math.-nat. Klasse am 12. März 2009 durch das w. M. August Florian)

\begin{abstract}
Assume that $n$ points are chosen independently and according to the uniform distribution from a convex polygon $C$. Consider the convex hull of the randomly chosen points. The probabilities $p_{k}^{(n)}(C)$ that the convex hull has exactly $k$ vertices are stated for all $k$ in the cases that $C$ is a square (equivalently a parallelogram) or a triangle.
\end{abstract}

Mathematics Subject Classification (2000): 52A22, 60D05.

Key words: Convex hull, random points, random polygons.

\section{Introduction}

The distribution of the number of vertices of the convex hull of $n$ points chosen independently and uniformly from a convex set is one of the oldest fields of research in geometrical probability, dating back to the sixties of the nineteenth century. In spite of the interest this topic has attracted throughout the years and in spite of the huge number of papers published on this and closely related topics, very little progress has been achieved in answering the original question where $n$ is a fixed number and $C$ a "simple" plane convex set like a square or a triangle. Even in these cases the probability $p_{k}^{(n)}(C)$ that the convex hull has 
exactly $k$ vertices is only known either if $n$ is very small, namely if $n \leq 6$, or if $k$ attains its minimal or maximal value, namely if $k=3$ or $k=n$, respectively; see [5] for references. For more information about the convex hull of random points see in particular the books by MATHAi [9] and SCHNEIDER and WeIL [12], as well as the surveys by AFFentranger [1], Buchta [3], Gruber [8], Schneider [10], [11], and WEIL and WIEACKER [13]. Many references are also contained in [2], [4], [6], and [7].

It was pointed out in [5] that the question of determining the distribution of the number of vertices of the convex hull of random points chosen from a convex polygon $C$ leads to the investigation of certain convex chains. More precisely, the probability $p_{k}^{(n)}(C)$ that the convex hull of $n$ points has exactly $k$ vertices can be expressed in terms of the probabilities $q_{j}^{(r)}(1 \leq j \leq k-2,1 \leq r \leq n-2)$ defined as follows:

Assume that $r$ points $P_{1}, \ldots, P_{r}$ are distributed independently and uniformly in the triangle with vertices $(0,1),(0,0)$, and $(1,0)$. Consider the convex hull of $(0,1), P_{1}, \ldots, P_{r}$, and $(1,0)$. The vertices of the convex hull form a convex chain. The probability that the convex chain consists - apart from the points $(0,1)$ and $(1,0)-$ of exactly $j$ of the points $P_{1}, \ldots, P_{r}$ is the required probability $q_{j}^{(r)}$.

The main result of [5] is the explicit formula

$$
q_{j}^{(r)}=2^{j} \sum \frac{i_{1} \cdots i_{j}}{i_{1}\left(i_{1}+1\right)\left(i_{1}+i_{2}\right)\left(i_{1}+i_{2}+1\right) \cdots\left(i_{1}+\cdots+i_{j}\right)\left(i_{1}+\cdots+i_{j}+1\right)},
$$

where the sum is taken over all $i_{1}, \ldots, i_{j} \in \mathbb{N}$ such that $i_{1}+\cdots+i_{j}=r$.

In the present note we state the arising formulae for $p_{k}^{(n)}(C)$ in the cases that $C$ is a square or a triangle. The proofs will be published in a future paper.

\section{Formulae for the Square}

Consider $n$ distinct points in a square $S$. For each edge the point with minimal distance to this edge is unique with probability one if the $n$ points are chosen independently and according to the uniform distribution. Assign to each edge the point with minimal distance. Mark the assigned points. Depending on whether

(a) each marked point is assigned to exactly one edge (such that the number of marked points is 4) or 
(b) one marked point is assigned to two adjacent edges, whereas each of the further marked points is assigned to exactly one of the remaining edges (such that the number of marked points is 3) or

(c) each marked point is assigned to two adjacent edges (such that the number of marked points is 2)

we say that the convex hull of the $n$ points is of type (a), (b), or (c), respectively.

The probabilities $p_{a}^{(n)}, p_{b}^{(n)}$, and $p_{c}^{(n)}$ that the convex hull is of type (a), (b), or (c), respectively, turn out to be

$$
p_{a}^{(n)}=\frac{(n-2)(n-3)}{n(n-1)}, \quad p_{b}^{(n)}=\frac{4(n-2)}{n(n-1)}, \quad \text { and } \quad p_{c}^{(n)}=\frac{2}{n(n-1)} .
$$

The vertices of the convex hull of the $n$ points are composed of the marked points and possibly of further points which are situated - in an obvious sense - "between" the marked points. The convex hull has $k$ vertices if the marked points - let us write $m$ for their number - and the further vertices between the marked points - let us write $k_{1}, \ldots, k_{m}$ for their respective numbers in counter-clockwise order - add up to $k$.

In order to derive the probabilities $p_{k \mid a}^{(n)}, p_{k \mid b}^{(n)}$, and $p_{k \mid c}^{(n)}$ that the convex hull of the $n$ random points, on condition that it is of type (a), (b), or (c), respectively, has exactly $k$ vertices, we first determine the probabilities $p_{k_{1} \ldots, k_{m}}^{(n)}$ that the convex hull has $k_{1}, \ldots, k_{m}$ vertices between the marked points, where $m=4,3$, or 2 depending on the type of the convex hull.

Whereas in the cases (a) and (c) it does not matter for symmetry reasons which marked point is assumed to be the first one, it does matter in case (b), and we denote the number of vertices between the two marked points which are assigned to only one edge by $k_{1}$.

The values of $p_{k_{1}, k_{2}, k_{3}, k_{4}}^{(n)}$ in case (a), of $p_{k_{1}, k_{2}, k_{3}}^{(n)}$ in case (b), and of $p_{k_{1}, k_{2}}^{(n)}$ in case (c) are obtained via polynomials associated with these probabilities: in case (a)

$$
\begin{aligned}
& P_{k_{1}, k_{2}, k_{3}, k_{4}}^{(n)}\left(x_{1}, x_{2}, x_{3}, x_{4}\right) \\
& =\frac{(n-4) !}{2^{n-4}} \sum_{\substack{r_{0}+r_{1}+\cdots+r_{4}=n-4 \\
r_{0} \geq 0 \\
r_{1} \geq k_{1}, \ldots, r_{4} \geq k_{4}}} \frac{1}{r_{0} !} A_{0}^{r_{0}} \prod_{i=1}^{4} \frac{1}{r_{i} !} q_{k_{i}}^{\left(r_{i}\right)} A_{i}^{r_{i}},
\end{aligned}
$$


where

$$
\begin{aligned}
& A_{0}=2-x_{1}-x_{2}-x_{3}-x_{4}+x_{1} x_{2}+x_{2} x_{3}+x_{3} x_{4}+x_{4} x_{1}, \\
& A_{1}=x_{1}\left(1-x_{2}\right), \\
& A_{2}=x_{2}\left(1-x_{3}\right), \\
& A_{3}=x_{3}\left(1-x_{4}\right), \\
& A_{4}=x_{4}\left(1-x_{1}\right),
\end{aligned}
$$

in case (b)

$$
\begin{aligned}
& P_{k_{1}, k_{2}, k_{3}}^{(n)}\left(x_{1}, x_{2}\right) \\
& =\frac{(n-3) !}{2^{n-3}} \sum_{\substack{r_{0}+r_{1}+r_{2}+r_{3}=n-3 \\
r_{0} \geq 0 \\
r_{1} \geq k_{1}, r_{2} \geq k_{2}, r_{3} \geq k_{3}}} \frac{1}{r_{0} !} B_{0}^{r_{0}} \prod_{i=1}^{3} \frac{1}{r_{i} !} q_{k_{i}}^{\left(r_{i}\right)} B_{i}^{r_{i}},
\end{aligned}
$$

where

$$
\begin{aligned}
& B_{0}=x_{1}+x_{2}-x_{1} x_{2}, \\
& B_{1}=x_{1} x_{2} \\
& B_{2}=1-x_{1} \\
& B_{3}=1-x_{2}
\end{aligned}
$$

and in case (c)

$$
P_{k_{1}, k_{2}}^{(n)}=\frac{(n-2) !}{2^{n-2}} \sum_{\substack{r_{1}+r_{2}=n-2 \\ r_{1} \geq k_{1}, r_{2} \geq k_{2}}} \prod_{i=1}^{2} \frac{1}{r_{i} !} q_{k_{i}}^{\left(r_{i}\right)} .
$$

The integration of these polynomials from 0 to 1 with respect to each variable yields the required probabilities: in case (a)

$$
p_{k_{1}, k_{2}, k_{3}, k_{4}}^{(n)}=\int_{0}^{1} \int_{0}^{1} \int_{0}^{1} \int_{0}^{1} P_{k_{1}, k_{2}, k_{3}, k_{4}}^{(n)}\left(x_{1}, x_{2}, x_{3}, x_{4}\right) d x_{1} d x_{2} d x_{3} d x_{4},
$$

in case (b)

$$
p_{k_{1}, k_{2}, k_{3}}^{(n)}=\int_{0}^{1} \int_{0}^{1} P_{k_{1}, k_{2}, k_{3}}^{(n)}\left(x_{1}, x_{2}\right) d x_{1} d x_{2}
$$


and in case (c)

$$
p_{k_{1}, k_{2}}^{(n)}=P_{k_{1}, k_{2}}^{(n)}
$$

As an obvious consequence we obtain in case (a)

$$
p_{k \mid a}^{(n)}=\sum_{\substack{k_{1}+\ldots+k_{4}=k-4 \\ k_{1} \geq 0, \ldots, k_{4} \geq 0}} p_{k_{1}, k_{2}, k_{3}, k_{4}}^{(n)},
$$

in case (b)

$$
p_{k \mid b}^{(n)}=\sum_{\substack{k_{1}+k_{2}+k_{3}=k-3 \\ k_{1} \geq 0, k_{2} \geq 0, k_{3} \geq 0}} p_{k_{1}, k_{2}, k_{3}}^{(n)}
$$

in case (c)

$$
p_{k \mid c}^{(n)}=\sum_{\substack{k_{1}+k_{2}=k-2 \\ k_{1} \geq 0, k_{2} \geq 0}} p_{k_{1}, k_{2}}^{(n)}
$$

and finally

$$
p_{k}^{(n)}(S)=p_{a}^{(n)} p_{k \mid a}^{(n)}+p_{b}^{(n)} p_{k \mid b}^{(n)}+p_{c}^{(n)} p_{k \mid c}^{(n)}
$$

The resulting values of $p_{k}^{(n)}(S)$ are new for $n \geq 7$ and $4 \leq k \leq n-1$. Table 1 displays for $n=7$ the products $p_{a}^{(7)} p_{k \mid a}^{(7)}, p_{b}^{(7)} p_{k \mid b}^{(7)}$, and $\bar{p}_{c}^{(7)} p_{k \mid c}^{(7)}$, i.e. the probabilities that the convex hull is of a certain type and has exactly $k$ vertices. The marginal probabilities in the last column are the probabilities that the convex hull is of type (a), (b), and (c), respectively, and the marginal probabilities in the last row are the required probabilities $p_{k}^{(7)}(S)$.

Table 1. Probabilities that the convex hull is of a certain type and has exactly $k$ vertices in the case that $C$ is a square and $n=7$

\begin{tabular}{c|ccccc|c}
\hline$k$ & 3 & 4 & 5 & 6 & 7 & $\sum$ \\
\hline$a$ & 0 & $\frac{65}{1008}$ & $\frac{155}{672}$ & $\frac{475}{3024}$ & $\frac{145}{6048}$ & $\frac{10}{21}$ \\
$b$ & $\frac{137}{10080}$ & $\frac{1811}{12600}$ & $\frac{1046}{4725}$ & $\frac{223}{2520}$ & $\frac{1361}{151200}$ & $\frac{10}{21}$ \\
$c$ & $\frac{1}{1008}$ & $\frac{437}{25200}$ & $\frac{1621}{75600}$ & $\frac{109}{15120}$ & $\frac{1}{1575}$ & $\frac{1}{21}$ \\
\hline$\sum$ & $\frac{7}{480}$ & $\frac{203}{900}$ & $\frac{3409}{7200}$ & $\frac{91}{360}$ & $\frac{121}{3600}$ & 1 \\
\hline
\end{tabular}




\section{Formulae for the Triangle}

Consider $n$ distinct points in a triangle $T$. If - analogously to the squareto each edge of the triangle the point with minimal distance is assigned and marked, either

(d) each marked point is assigned to exactly one edge (such that the number of marked points is 3 ) or

(e) one marked point is assigned to two adjacent edges and a further marked point is assigned to the remaining edge (such that the number of marked points is 2),

and we say that the convex hull is of type (d) or (e), respectively.

For the probabilities $p_{d}^{(n)}$ and $p_{e}^{(n)}$ that the convex hull is of type (d) or (e), respectively, we find that

$$
p_{d}^{(n)}=\frac{2(n-2)}{2 n-1} \quad \text { and } \quad p_{e}^{(n)}=\frac{3}{2 n-1} .
$$

The probabilities $p_{k \mid d}^{(n)}$ and $p_{k \mid e}^{(n)}$ are defined analogously to the square. The values of $p_{k_{1}, k_{2}, k_{3}}^{(n)}$ in case (d) and of $p_{k_{1}, k_{2}}^{(n)}$ in case (e) are again obtained via polynomials associated with these probabilities: in case (d)

$$
\begin{aligned}
& P_{k_{1}, k_{2}, k_{3}}^{(n)}\left(x_{1}, x_{2}, x_{3}\right) \\
& =(n-3) ! \sum_{\substack{r_{0}+r_{1}+r_{2}+r_{3}=n-3 \\
r_{0} \geq 0 \\
r_{1} \geq k_{1}, r_{2} \geq k_{2}, r_{3} \geq k_{3}}} \frac{1}{r_{0} !} D_{0}^{r_{0}} \prod_{i=1}^{3} \frac{1}{r_{i} !} q_{k_{i}}^{\left(r_{i}\right)} D_{i}^{r_{i}},
\end{aligned}
$$

where

$$
\begin{aligned}
& D_{0}=1-x_{1}-x_{2}-x_{3}+x_{1} x_{2}+x_{2} x_{3}+x_{3} x_{1}, \\
& D_{1}=x_{1}\left(1-x_{2}\right) \\
& D_{2}=x_{2}\left(1-x_{3}\right) \\
& D_{3}=x_{3}\left(1-x_{1}\right)
\end{aligned}
$$

and in case (e)

$$
P_{k_{1}, k_{2}}^{(n)}\left(x_{1}\right)=(n-2) ! \sum_{\substack{r_{1}+r_{2}=n-2 \\ r_{1} \geq k_{1}, r_{2} \geq k_{2}}} \prod_{i=1}^{2} \frac{1}{r_{i} !} q_{k_{i}}^{\left(r_{i}\right)} E_{i}^{r_{i}}
$$


Table 2. Probabilities that the convex hull is of a certain type and has exactly $k$ vertices in the case that $C$ is a triangle and $n=7$

\begin{tabular}{c|ccccc|c}
\hline$k$ & 3 & 4 & 5 & 6 & 7 & $\sum$ \\
\hline$d$ & $\frac{31}{2340}$ & $\frac{181}{975}$ & $\frac{12761}{35100}$ & $\frac{322}{1755}$ & $\frac{409}{17550}$ & $\frac{10}{13}$ \\
$e$ & $\frac{1}{39}$ & $\frac{92}{975}$ & $\frac{497}{5850}$ & $\frac{14}{585}$ & $\frac{11}{5850}$ & $\frac{3}{13}$ \\
\hline$\sum$ & $\frac{7}{180}$ & $\frac{7}{25}$ & $\frac{1211}{2700}$ & $\frac{28}{135}$ & $\frac{17}{675}$ & 1 \\
\hline
\end{tabular}

where

$$
\begin{aligned}
& E_{1}=x_{1}, \\
& E_{2}=1-x_{1} .
\end{aligned}
$$

The integration of these polynomials from 0 to 1 with respect to each variable yields the required probabilities. Everything else is analogous to the square.

As above the resulting values of $p_{k}^{(n)}(T)$ are new for $n \geq 7$ and $4 \leq k \leq n-1$. Table 2 displays for $n=7$ the products $p_{d}^{(7)} p_{k \mid d}^{(7)}$ and $p_{e}^{(7)} p_{k \mid e}^{(7)}$, i.e. the probabilities that the convex hull is of a certain type and has exactly $k$ vertices. The marginal probabilities in the last column are the probabilities that the convex hull is of type (d) and (e), respectively, and the marginal probabilities in the last row are the required probabilities $p_{k}^{(7)}(T)$.

\section{Acknowledgement}

The author would like to thank Dr. MARIA ALICE BERTOLIM for carefully checking the values displayed in Tables 1 and 2 .

\section{References}

[1] Affentranger, F. (1992) Aproximación aleatoria de cuerpos convexos. Publ. Mat. Barc. 36: 85-109

[2] BÁRÁNY, I., BuChTA, C. (1993) Random polytopes in a convex polytope, independence of shape, and concentration of vertices. Math. Ann. 297: 467-497

[3] BuchTA, C. (1985) Zufällige Polyeder - Eine Übersicht. In: HLAwKA, E. (ed.) Zahlentheoretische Analysis, pp. 1-13. Lecture Notes in Mathematics, Vol. 1114, Springer, Berlin

[4] BUCHTA, C. (2005) An identity relating moments of functionals of convex hulls. Discrete Comput. Geom. 33: 125-142

[5] BuchTA, C. (2006) The exact distribution of the number of vertices of a random convex chain. Mathematika 53: 247-254 
[6] Buchta, C., ReitzNer, M. (1997) Equiaffine inner parallel curves of a plane convex body and the convex hulls of randomly chosen points. Probab. Theory Relat. Fields 108: $385-415$

[7] Buchta, C., Reitzner, M. (2001) The convex hull of random points in a tetrahedron: Solution of Blaschke's problem and more general results. J. Reine Angew. Math. 536: 1-29

[8] GRUBER, P. M. (1997) Comparisons of best and random approximation of convex bodies by polytopes. Rend. Circ. Mat. Palermo (2) Suppl. 50: 189-216

[9] MathaI, A. M. (1999) An Introduction to Geometrical Probability. Distributional Aspects with Applications. Gordon and Breach, Amsterdam

[10] SCHNEIDER, R., (1988) Random approximation of convex sets. J. Microscopy 151: $211-227$

[11] SCHNEIDER, R., (2004) Discrete aspects of stochastic geometry. In: GOODMAN, J. E., O'Rourke, J. (eds.) Handbook of Discrete and Computational Geometry, 2nd edn., pp. 255-278. Chapman and Hall/CRC, Boca Raton, Florida

[12] SchneIDER, R., WeIL, W. (2008) Stochastic and Integral Geometry. Springer, Berlin

[13] Weil, W., Wieacker, J. A. (1993) Stochastic geometry. In: Gruber, P. M., Wills, J. M. (eds.) Handbook of Convex Geometry, Vol. B, pp. 1391-1438. North-Holland/Elsevier, Amsterdam

Author's address: Prof. Dr. Christian Buchta, Fachbereich Mathematik, Universität Salzburg, Hellbrunner Straße 34, A-5020 Salzburg, Austria. E-Mail: christian.buchta@sbg.ac.at. 